\title{
Predictive accuracy of semi-quantitative scoring to screen for unfavorable ejection fraction and infarct size
}

\author{
Lara Bakhos", Maria M Izquierdo-Gomez, Daniel C Lee, Edwin Wu \\ From 2011 SCMR/Euro CMR Joint Scientific Sessions \\ Nice, France. 3-6 February 2011
}

\section{Background}

Manual planimetry for quantitative analysis of ejection fraction (EF) and infarct size (IS) on cardiac magnetic resonance (CMR) imaging are too time-consuming, and therefore, impractical in daily clinical practice. We sought to derive and examine the predictive accuracy of a semi-quantitative scoring technique to screen patients with an EF $\leq 35 \%$ or an IS $\geq 18.5 \%$, known independent predictors of increased cardiac events and mortality.

\section{Methods}

The CMR derivation cohort consisted of 122 STsegment elevation myocardial infarction patients. The validation cohort consisted of an additional 172 patients from the multi-center DEfibrillators To REduce Risk by MagnetIc ResoNance Imaging Evaluation (DETERMINE) trial. Cines were scored on a 17-segment model for wall motion and totaled for the Sum Motion Score (SMS): 0 = normal, 1 = mild hypokinesis, $2=$ moderate to severe hypokinesis, $3=$ akinesis, $4=$ dyskinesis. Viability images were scored for infarct transmurality and totaled for the Sum Infarct Score (SIS): $0=$ none, $1=$ $1-25 \%, 2=26-50 \%, 3=51-75 \%, 4=76-100 \%$. Quantitative EF and IS were manually planimetered using QMass (Medis).

\section{Results}

From the derivation cohort, the SMS correlated with EF $(\mathrm{R}=-0.91, \mathrm{p}<0.001)$ and SIS correlated with IS $(\mathrm{R}=0.94$, $\mathrm{p}<0.001)$. Linear regression equations between SMS vs. $\mathrm{EF}$ and SIS vs. IS were obtained to estimate EF (est$\mathrm{EF}=55$ - SMS) and IS (est-IS $=1.383 *$ SIS).
In the validation cohort, the mean EF was $39.0 \pm$ $11.7 \%$ (32\% with $\mathrm{EF} \leq 35 \%$ ), and the mean IS was $17.3 \pm$ $10.4 \%$ ( $40 \%$ with IS $\leq 18.5 \%$ ). Using the derivation formula, the est-EF $(36.2 \pm 10.9 \%)$ correlated with $E F$ $(\mathrm{R}=0.9)$ with a slight underestimation of the mean difference by $2.8 \pm 4.9 \%$. In addition, the est-IS $(21.8 \pm$ $12.8 \%)$ correlated with IS $(\mathrm{R}=0.9)$. The est-IS tended to overestimate IS by $4.5 \pm 5.9 \%$. The sensitivity for detecting an $\mathrm{EF} \leq 35 \%$ using the est-EF was $94 \%$ with a negative predictive value of $97 \%$. The sensitivity for detecting an IS $\geq 18.5 \%$ using the est-IS was $96 \%$ with a negative predictive value of $96 \%$.

\section{Conclusions}

Semi-quantitative scoring is a sensitive screening tool that can be used to identify patients with an ejection fraction $\leq 35 \%$ or infarct size $\geq 18.5 \%$ to provide a rapid alternative method to manual planimetry.

Published: 2 February 2011

doi:10.1186/1532-429X-13-S1-P44

Cite this article as: Bakhos et al:: Predictive accuracy of semi-

quantitative scoring to screen for unfavorable ejection fraction and

infarct size. Journal of Cardiovascular Magnetic Resonance 2011 13(Suppl 1): P44. 